\title{
LATE-BREAKING POSTERS
}

68

\section{The most common physical disorders after subarachnoidal hemorrhage with patients with no previous diagnosis of AV malformation or Aneurysm V. Vargek-Solter, M. Ivica, V. Vrabec Barta, D. Jurišić \& V. Demarin University Department of Neurology, Sestre Milosrdnice University Hospital, Reference Center for Neurovascular Disorders and Reference Center for Headache of the Ministry of Health and Social Welfare of the Republic of Croatia, Vinogradska 29, Zagreb, Croatia}

The rupture of aneurysm and subarachnoidal hemorrhage (SAH) is complex intracranial condition. Often accompanied with dramatic clinical presentation, necessity for emergency diagnostic treatment and various complications. To make sure that proper diagnostic approach is provided for this patients University Department of Neurology, University Hospital "Sestre milosrdnice", Croatian Society of Physicians, Croatian Society of Stroke Prevention, Croatian Society of Cerebrovascular Diseases of Croatian Society of Physicians issued references for treatment patients with stroke. Five-year study analysis of patients with clinical presentation of SAH at University Department of Neurology, University Hospital "Sestre milosrdnice" monitored the most common physical disorders with patients who had CT verified SAH, but no previously verified diagnosis of AV malformation or aneurysm. We analyzed EKG parameters, especially ST-T segment, alterations of QRS complex and ST segment. Preliminary results showed that EKG alterations spontaneously recovered in the first days after diagnosing $\mathrm{SAH}$ and were no predictors of outcome. Forward, we analyzed and some laboratory parameters, especially sodium level in blood. Hyponatriemia often appeared with SAH patients that can be explained by disruption of blood brain barrier and accumulation of sodium and interstitial fluid in intercellular space.

69

\section{Hypergycemia in subarachnoid hemorrhage}

Supanc Višnja, Breitenfeld Tomislav, Vargek-Solter Vesna, Ivica Marija \& Demarin Vida

University Department of Neurology, Sestre Milosrdnice University

Hospital, Reference Center for Neurovascular Disorders and

Reference Center for Headache of the Ministry of Health and Social Welfare of the Republic of Croatia, Vinogradska 29, Zagreb, Croatia

Background: Hyperglycemia after subarachnoid hemorrhage (SAH) is common, but extend to which it contributes to inhospital complications is still unknown The aim of this study was to determine correlation between level of blood glucose with the clinical presentation of subarachnoid hemorrhage described by the Hunt and Hess scale.

Methods and patients: We examined a cohort of SAH patients who had at least 3 daily blood glucose measurements between SAH diagnosed day 0 and day 10 . We also defined mean glucose burden $(\mathrm{GB})$ as the average peak daily blood glucose level $>6.0 \mathrm{mmol} / \mathrm{L}$ and hospital complications were recorded prospectively.

Results: Our study included 89 patients with diagnose SAH between the period 2003 and 2008, $42(47 \%)$ of them where men and $47(53 \%)$ were women. A $63(71 \%)$ of patients had peak daily glucose level $>6 \mathrm{mmol} / \mathrm{L}$. The blood glucose level (BGL) $6-$ $7 \mathrm{mmol} / \mathrm{L}$ had $35(55 \%)$ patients, 18 with HH I, 7 with HH II, 6 with $\mathrm{HH} 0,3$ with HH III and 1 with HH V. The 17 (27\%) patients with BGL $8-9.9 \mathrm{mmol} / \mathrm{L}$ had mostly HH I, 8 of them, HH II-III had 6 patients, 3 had HH IV-V. In the group BLG 10-11.9 mmol/L were $5(8 \%)$ patients, 4 of them with $\mathrm{HH} \mathrm{I}$ and $\mathrm{HH}$ III, 1 with $\mathrm{HH}$ V. Among the $6(9.5 \%)$ patients with BGL over $12 \mathrm{mmol} / \mathrm{L}, 3$ of them had HH V and 3 had HH I. Among 63 patients 7 (11\%) died, 5 with HH V, 1 with HH III and BGL 10-11.9 mmol/L and 1 with HH I and BGL $8-9.9 \mathrm{mmol} / \mathrm{L}$.

Conclusions: Predictors of high-GB included eldery patients, Hunt and Hess grade III-V and history of diabetes mellitus. The GB was associated with increased intensive care unit length of stay, congestive heart failure, respiratory failure and brain stem compression from herniation. We can conclude that hypergycemia after $\mathrm{SAH}$ is associated with serious hospital complications.

\section{0}

\section{Some epidemiologic characteristics of multiple sclerosis patients \\ Bošnjak Pašić M. ${ }^{1}$, Podobnik Šarkanji S. ${ }^{2}$, Dežmalj Grbelja L. ${ }^{1}$ \& V. Demarin ${ }^{1}$ \\ ${ }^{1}$ University Department of Neurology, Sestre Milosrdnice University Hospital, Zagreb, Croatia, ${ }^{2}$ Clinic Podobnik, Zagreb, Croatia}

Multiple sclerosis (MS) is chronic inflammatory autoimmune demyelinative disease of CNS. In most patients first symptoms of the disease appear at the age between 20 and 45 years. Females are two times more affected and some geographic regions have higher risk. MS can have benign or malignant course which can be: relapsing-remitting MS (RRMS), secondary progressive MS (SPMS), primary progressive MS (PPMS) and progressive-relapsing MS (PRMS). The most common subtype, in about $85 \%$ patients, is RRMS.

In our investigation we interviewed 100 randomized patients, who have diagnosis of MS for different long period. We analized some general characteristics (age, sex, profession, married state, child number, some data from personal and familiar anamnesis), type of treatment, type of disease, present health state and quality of life. As it is characteristic for MS, patients were in young or middle age, in most patients first signs of disease were unrecognized at the beginning, some symptoms were present before the diagnosis was established. First symptoms were subjective sensory disturbances, visual impairment, weakness of one or more extremities, diplopia, vertigo, drowsiness, bladder and bowel dysfunction. The most frequent type of treatment is pulse corticosterid therapy in relapse, polyvitamins and physical therapy later. Minor number of patients takes other type of treatment as beta interferon.

As the disease is connected with worsening of disability during the time, some patients are not capable for work, and earlier pensioning can not be avoided.

If our investigation would continue in a greater number inteviewed patients, we could have a basis of data with significant characteristics of the disease in our region. 